\title{
Ballistic impact response of steel fiber-metal laminates
}

\author{
George Bikakis ${ }^{1,2 *}$, Nikolaos Tsigkros ${ }^{2}$, Emilios Sideridis ${ }^{2}$, and Alexander Savaidis ${ }^{1}$ \\ ${ }^{1}$ School of Pedagogical and Technological Education, Mechanical Engineering Educators \\ Department, GR 14121 N. Heraklion, Athens, Greece \\ ${ }^{2}$ National Technical University of Athens, Strength of Materials Laboratory, GR 15773 Zographou, \\ Athens, Greece
}

\begin{abstract}
In this article, the ballistic impact response of square clamped fiber-metal laminates and monolithic plates consisting of different metal alloys is investigated using the ANSYS LS-DYNA explicit nonlinear analysis software. The panels are subjected to central normal high velocity ballistic impact by a cylindrical projectile. Using validated finite element models, the influence of the mechanical properties of the constituent metal alloy on the ballistic resistance of the fiber-metal laminates and the monolithic plates is studied. Six steel alloys are examined, namely 304 stainless steel, 1010, 1080, 4340, A36 steel and DP 590 dual phase steel. A comparison with the response of GLARE plates is also implemented. It is found that the ballistic limits of the panels can be substantially affected by the constituent alloy. The stainless steel based panels offer the highest ballistic resistance followed by the A36 steel based panels which in turn have higher ballistic resistance than the 2024-T3 aluminum based panels. The A36 steel based panels have higher ballistic limit than the 1010 steel based panels which in turn have higher ballistic limit than the 1080 steel based panels. The behavior of characteristic impact variables during the ballistic impact phenomenon is analyzed.
\end{abstract}

\section{Introduction}

Fiber-metal laminates are hybrid composite materials, consisting of alternating metal layers bonded to fiber-reinforced prepreg layers. ARALL (Aramid Reinforced ALuminum Laminates), CARALL (CArbon Reinforced ALuminum Laminates) and GLARE (GLAss REinforced) belong to this new family of materials. Apart from aluminum, other metal constituents such as steel, magnesium and titanium have been employed in order to manufacture fiber-metal laminates. The possible variations of hybrid laminated material systems are numerous and their mechanical evaluation is a challenging intensive field of scientific research.

The response of steel based fiber-metal laminates under various loading conditions is very interesting since steel is a classical construction material and their applications can be numerous. Recently, scientific articles concerning fiber-metal laminates with steel metal

\footnotetext{
${ }^{*}$ Corresponding author: bikakis.george@yahoo.com
} 
layers have been published [1-9]. In these articles, various applications are considered: thick walled tubes subjected to axisymmetric internal pressure [9], shear walls for the construction of buildings [6], panels for underwater applications [5] and industrial applications [4]. The mechanical evaluation of steel based fiber-metal laminates includes their impact response, fatigue characteristics, creep behavior, hygrothermal conditioning, response to earthquake loading [1-9].

The design of impact resistant laminated structures is very important for industries such as aerospace, automotive and shipping since during the life of a structure, impact by foreign objects can be expected to occur during manufacturing, service and maintenance operations. Furthermore, the design of laminated armor to defeat small-caliber projectiles is of primary interest in military applications.

In this article, extensive research has been implemented in order to investigate and compare the influence of the mechanical properties of different steel alloys on the ballistic resistance of glass-reinforced fiber-metal laminates and monolithic metal plates consisting of these alloys. ANSYS LS-DYNA finite element software is employed for this purpose. A total of 30 different ballistic impact cases have been simulated. Furthermore, the behavior of characteristic impact variables during the impact event is studied. The presented results shed light to the ballistic impact response of steel plates, GLARE plates and steel based fiber-metal laminates and will help engineers and researchers to understand the behavior of the examined materials along with the effect of using different metal alloy for their manufacturing. No other evaluation of the ballistic resistance of fiber-metal laminates consisting of the 6 steel alloys examined in this article is known to the authors.

\section{Finite element modeling}

In order to simulate the normal ballistic impacts of free-flying projectiles on the targets, we use the structural arrangement of the ballistic impact experiments of reference [10]. In our finite element modeling procedure we employ SOLID164 elements for the meshing of the projectile and the targets.

In order to simulate the contact between the projectile and the panel we use the surfaceto-surface contact type with the eroding contact option. For the simulation of the contact between adjacent layers of the fiber-metal laminate we use the surface-to-surface contact type with the tiebreak contact option. The tiebreak contact option is needed in order to allow delamination/debonding between adjacent layers.

In order to reduce the computational cost, the projectile is considered rigid. For this reason, a rigid material model is used for the projectile. The simplified Johnson-Cook plasticity material model [11] is employed for modeling the material behavior of aluminum. We idealize the material behavior of the unidirectional glass-epoxy layers in the fiber-metal laminates by employing the Chang-Chang composite failure model [11].

For the simulation of the perforation of the panels, the panels' finite elements in way of the projectile must be eroded. In order to achieve this goal, a strain-based erosion criterion is added to the metal and glass-epoxy material models.

An issue that needs special attention is the determination of the in-plane material axes of the prepreg layers, since their material properties correspond to the material coordinate system. The ANSYS LS-DYNA volume brick orient command has been employed in order to specify the solid brick element orientation for the volume mapped (hexahedron) meshing. It is noted that in our modeling procedure, each prepreg layer corresponds to a separate volume. The fiber directions determine the orientation of the material coordinate system. In ANSYS LS-DYNA by default, the material coordinate system is parallel to the element coordinate system which is oriented in accordance with the direction of the fibers, using the aforementioned volume brick orient command. 
In order to further reduce the high computational cost of the considered problem, we take advantage of the symmetry of the problem by modeling a quarter of the structure and by applying suitable symmetry boundary conditions to all nodes of the symmetry planes.

Given that the projectile is considered rigid, instead of meshing a solid cylinder, only the exterior surface with $0.8 \mathrm{~mm}$ thickness was meshed. The density of the projectile's material was modified so that the full projectile's mass remains constant. A fine mesh is used for the projectile in order to represent its geometry accurately, including the radius of its impacting face. With this meshing approach the number of the finite elements needed to model the projectile is minimized and the computational cost is further reduced. In order to verify the accuracy of the hollow projectile model FEM results, complete projectile models were also implemented and solved yielding practically identical results.

The one-point integration has been selected for the SOLID164 elements. For each ballistic impact case we analyze, we verify that the hourglass energy and the sliding energy are small relative to the internal energy, as recommended for an explicit analysis with LSDYNA [12].

An explicit transient dynamic analysis is employed with geometric and material nonlinearities. The initial velocity of the projectile is predetermined. The duration of our analysis is controlled with a predetermined termination time which allows for complete panel perforation, provided that the initial velocity of the projectile is greater than or equal to the ballistic limit. It is noted that in order to determine the unknown ballistic limit of a specific panel, several trial analyses have to be executed with different initial projectile velocity.

In order to verify the convergence of FEM results, the ballistic limit of the panel, we built two models with increasing in-plane mesh density for each specific case of square panel we analyze. The projectile's mesh density remains the same for all models. For each model we obtain the ballistic limit and compare them in order to verify that satisfactory convergence has been achieved.

It is noted that the finite element models of this article have also been used in reference [13] in order to predict the ballistic impact response of fiber-metal laminates and monolithic metal plates consisting of different aluminum alloys, and by comparison with published experimental data the models were validated there.

\section{Results and discussion}

Using the five aforementioned validated finite element models [13], we study the effect of the mechanical properties of different commercially available steel alloys on the ballistic resistance of steel based fiber-metal laminates and monolithic steel plates. Fiber-metal laminates and monolithic metal plates consisting of 304 stainless steel, 1010, 1080, 4340, A36 steel alloys and DP 590 dual phase steel are examined. These alloys are used in a wide variety of applications such as automotive, ship, aerospace and military structures, buildings, oil rigs, bridges and industrial applications. For this purpose, the mechanical properties of the initial 2024-T3 aluminum material of the panels were updated in each validated model according to Table 1 . This approach was also implemented in reference [14] in order to investigate the influence of the properties of aluminum alloys on the lowimpulse blast behavior of fiber-metal laminates. It is noted that the ballistic limit convergence along with the desired low levels of hourglass and sliding energy were verified in all of the 30 modeled ballistic impact cases. 
Table 1. Material properties and Johnson-Cook constants of steel alloys

\begin{tabular}{lllllll}
\hline $\begin{array}{l}\text { Steel } \\
\text { Alloy: }\end{array}$ & $\begin{array}{c}304 \\
\text { stainless }\end{array}$ & 1010 & 1080 & 4340 & A36 & DP 590 \\
\hline$\rho\left(\mathrm{kg} / \mathrm{m}^{3}\right)$ & $7800^{[15]}$ & $7870^{[16]}$ & $7700^{[16]}$ & $7830^{[19]}$ & $7850^{[16]}$ & $7870^{[21]}$ \\
$v$ & $0.265^{[16]}$ & $0.300^{[16]}$ & $0.270^{[18]}$ & $0.290^{[19]}$ & $0.260^{[16]}$ & $0.300^{[21]}$ \\
$\mathrm{E}(\mathrm{GPa})$ & $200.0^{[15]}$ & $200.0^{[16]}$ & $202.8^{[18]}$ & $200.0^{[19]}$ & $200.0^{[16]}$ & $214.0^{[21]}$ \\
$\mathrm{A}(\mathrm{MPa})$ & $310.00^{[15]}$ & $367.00^{[17]}$ & $525.00^{[18]}$ & $792.00^{[19]}$ & $286.13^{[20]}$ & $430.00^{[22]}$ \\
$\mathrm{B}(\mathrm{MPa})$ & $1000.00^{[15]}$ & $700.00^{[17]}$ & $3590.00^{[18]}$ & $510.00^{[19]}$ & $500.14^{[20]}$ & $823.60^{[22]}$ \\
$\mathrm{n}$ & $0.6500^{[15]}$ & $0.9350^{17]}$ & $0.6677^{[18]}$ & $0.2600^{[19]}$ & $0.2280^{[20]}$ & $0.5071^{[22]}$ \\
$\mathrm{C}$ & $0.0700^{[15]}$ & $0.0450^{[17]}$ & $0.0290^{[18]}$ & $0.0140^{[19]}$ & $0.0170^{[20]}$ & $0.0171^{[22]}$ \\
\hline
\end{tabular}

In Figure 1 the calculated ballistic limits of the two monolithic plates with $1.6 \mathrm{~mm}$ and $3.2 \mathrm{~mm}$ thickness are compared. As the thickness of the two examined plates increases, the corresponding ballistic limit is increased for all alloys. It can be observed from Figure 1 that the trend of the ballistic limits versus the panel thickness is similar for the considered alloys. Another observation from this figure is that the 304 stainless steel has greater ballistic resistance than the A36 mild steel and the 2024-T3 aluminum has lower ballistic resistance than the A36 mild steel. Similar pertinent experimental results have been reported in reference [23]. In that work mild steel, stainless steel and aluminum plates have been tested at several plates thicknesses. They were impacted by blunt cylindrical projectiles in order to determine their ballistic limit. It can be verified for plate thicknesses equal to $1.6 \mathrm{~mm}$ and $3.2 \mathrm{~mm}$ that the stainless steel offers the highest whereas the aluminum offers the lowest ballistic resistance among the three materials according to the experiments of Corran et al. as well. It can be observed from [23] that within this range of plate thickness, the experimental ballistic limit of the three materials varies approximately linearly versus the plate thickness. Consequently, it is very useful to present linear equation trendlines of ballistic limits as a function of the plate thickness for the materials examined here. These approximation equations are given in Figure 1 and are applicable for plate thickness values between $1.6 \mathrm{~mm}$ and $3.2 \mathrm{~mm}$. They can be used when experimental or theoretically calculated ballistic limits are not available for a specific thickness value.

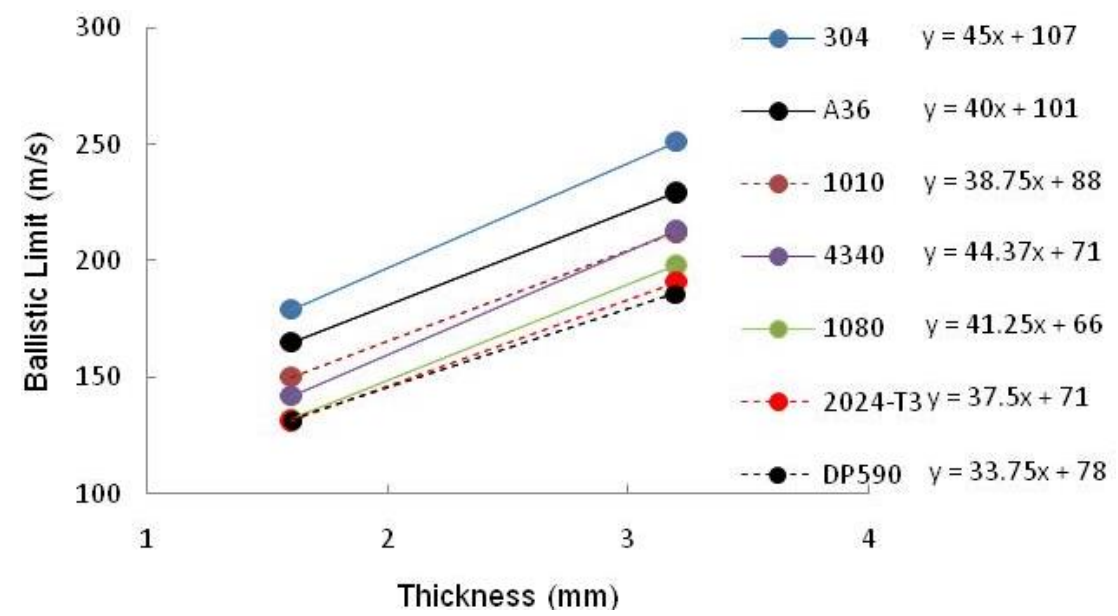

Fig. 1. Ballistic limits and trendlines of monolithic plates consisting of different metal alloys versus the plate thickness. 
It can be seen from Figure 1 that A36 steel offers higher ballistic limit than 1010 steel. Referring to the $1.6 \mathrm{~mm}$ thick plates, the calculated ballistic limits are $165 \mathrm{~m} / \mathrm{s}$ and $150 \mathrm{~m} / \mathrm{s}$, respectively. There are several empirical formulae to predict the critical impact energy required to perforate steel plates subjected to impact loading by projectiles [24]. In order to compare the aforementioned ballistic limits, we have applied the Jowett's formulae [24], which are based on experimental data. These relationships are suitable for impact on steel plates by short cylindrical projectiles. The minimum energy required for perforation is expressed as a function of the ultimate stress of steel, the diameter of the projectile, the thickness of the target, and the span of the target. Jowett's formulae have a specific validity range. Using Jowett's formulae we have calculated the ballistic energy and the corresponding ballistic velocity for the A36 steel and 1010 steel plates with $1.6 \mathrm{~mm}$ thickness. Only these two cases of our study satisfy all restrictions concerning the validity range of Jowett's formulae. Considering UTS values equal to $483 \mathrm{MPa}$ for the A36 steel [16] and $365 \mathrm{MPa}$ for the 1010 steel [16], the empirical ballistic velocities are found to be $151.3 \mathrm{~m} / \mathrm{s}$ and $131.5 \mathrm{~m} / \mathrm{s}$, respectively. Consequently, A36 steel offers higher ballistic limit than 1010 steel according to the empirical formulae as well. Furthermore, the empirical ballistic limits deviate $8.3 \%$ and $12.3 \%$ from their numerical values, respectively. This comparison further demonstrates the validity of the implemented finite element modeling procedure.

In Figure 2 the calculated ballistic limits of the fiber-metal laminates are compared. It is seen from this figure that the ballistic limit of the examined $2 / 1-0.5,3 / 2-0.3$ and $3 / 2-0.5$ fiber metal laminates increases versus the total panel thickness following a similar trend regardless of the monolithic metal constituent. Second order polynomial trendlines interpolate the published experimental and numerical data [10,13] concerning the ballistic limits of three GLARE 5 plates very smoothly. Such trendlines are employed in Figure 2 as well. A smooth interpolation of the calculated ballistic limits can be observed in Figure 2. Consequently, it is very useful to present second order polynomial equation trendlines of ballistic limits as a function of the panel thickness for the materials examined here. These approximation equations are given in Figure 2 and are applicable for panel thickness values between $1.52 \mathrm{~mm}$ and $2.54 \mathrm{~mm}$. They can be used when experimental or theoretically calculated ballistic limits are not available for a specific thickness value.

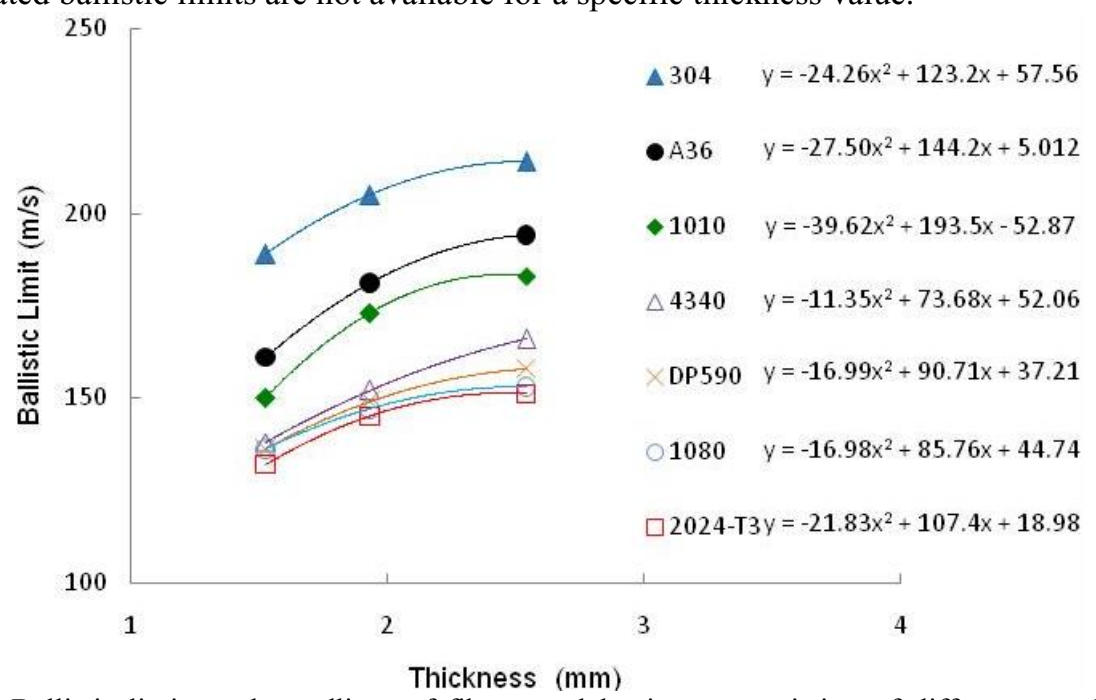

Fig. 2. Ballistic limits and trendlines of fiber-metal laminates consisting of different metal alloys versus the panel thickness.

It is seen from Figure 2 that the stainless steel based fiber-metal laminates offer higher 
ballistic resistance than the A36 steel based fiber-metal laminates which in turn have higher ballistic resistance than the GLARE panels. It is also seen that A36 steel based fiber-metal laminates have higher ballistic limit than 1010 steel based fiber-metal laminates. Furthermore, from Figures 1 and 2 it can be verified that the 1010 steel based panels offer higher ballistic limit than the 1080 steel based panels. Consequently, taking also into account the discussed behavior of monolithic plates consisting of 304 stainless steel, A36 and 1010 steel, and 2024-T3 aluminum, it is found that the behavior of fiber-metal laminates is substantially affected by the behavior of their metal constituent. This finding means that the impact resistance of fiber-metal laminates can be improved by selection of an impact tolerant metal alloy. Another observation from Figures 1 and 2 is that the 2024T3 aluminum, the 1080 steel and the DP 590 dual phase steel based panels have comparable relatively low ballistic resistance. From Figures 1 and 2 it is also concluded that the panel thickness is another important design parameter which can be used in order to increase the ballistic limit of fiber-metal laminates and monolithic plates.

In Figure 3 a representative diagram of the variation of characteristic impact variables versus time is shown. This diagram corresponds to a 1080 steel based fiber-metal laminate with 3/2-0.5 layup subjected to ballistic impact. Specifically, the time histories of the projectile's displacement, velocity and kinetic energy, symbolized with $w, u$ and $E_{k}$, respectively, are plotted along with the time history of the energy absorbed by the panel, $E_{a b}$. The $(w, t)$ and $(u, t)$ curves are directly obtained from the FEM analysis results. The $\left(E_{k}\right.$ , t) curve is calculated using the relation $E_{k}=0.5 \mathrm{mu}^{2}$. The sum of the projectile's kinetic energy and the panel's absorbed energy is equal to the total energy of the system which remains constant at the level of the initial kinetic energy of the projectile.

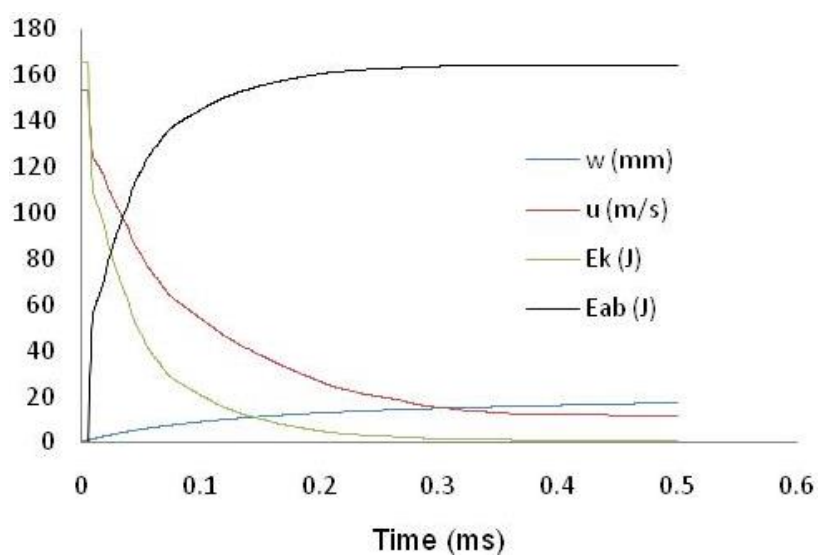

Fig. 3. Striker-target time history curves of a 1080 steel based 3/2-0.5 fiber-metal laminate subjected to ballistic impact by a cylindrical projectile.

By observation of Figure 3 it can be seen that the displacement of the projectile is initially equal to zero (for $\mathrm{t}=0$ ) and starts increasing with decreasing rate due to the panel's resistance. When the panel is perforated, about $0.37 \mathrm{~ms}$ after the beginning of the striker's motion, its resisting force acting on the projectile becomes equal to zero and the projectile's displacement increases with constant rate since it now moves with constant velocity according to Newton's first law.

It is seen from Figure 3 that the striker's ballistic velocity is initially equal to $153 \mathrm{~m} / \mathrm{s}$ and decreases abruptly because of the impact load applied on the projectile during its contact interaction with the clamped panel. The drop rate of the projectile's velocity becomes gradually lower as the striker penetrates the target and its stiffness is reduced. The onset and development of plasticity in the metal layers, the delamination between adjacent 
layers, the failure of composite layers and the erosion of each layer contribute to the reduction of the panel's stiffness. Eventually the panel is perforated and the projectile then continues to move but with constant low velocity as discussed previously.

It can be observed from Figure 3 that the ballistic kinetic energy of the projectile is initially equal to $165.3 \mathrm{~J}$ and is decreased abruptly since it is consumed to deform, cause damage and perforate the panel. After complete perforation the striker's kinetic energy becomes practically equal to zero. This means that the initial impact energy is consumed during the ballistic impact of the panel. The energy absorbed by the panel follows the opposite trend.

In Figure 4 the $(\mathrm{u}, \mathrm{w}),\left(\mathrm{E}_{\mathrm{k}}, \mathrm{w}\right)$ and $\left(\mathrm{E}_{\mathrm{ab}}, \mathrm{w}\right)$ curves corresponding to the time histories of Figure 3 are depicted. Since the projectile is initially (for $t=0$ ) located at a distance of 1 $\mathrm{mm}$ from the panel, it can be observed from Figure 4 that complete perforation occurs when the projectile is displaced about $14 \mathrm{~mm}$ from the position of its initial contact with the panel. Given that the total thickness of the clamped panel is $2.54 \mathrm{~mm}$, it is found that the panel has experienced large lateral deflections and significant membrane stretching before perforation occurs. The behavior of the considered impact variables which is depicted in Figures 3 and 4 is a useful contribution for the understanding of the examined ballistic impact phenomenon. To the authors' knowledge, there is not any published literature that demonstrates how these variables vary as a function of time and displacement during the ballistic impact event of fiber-metal laminates.

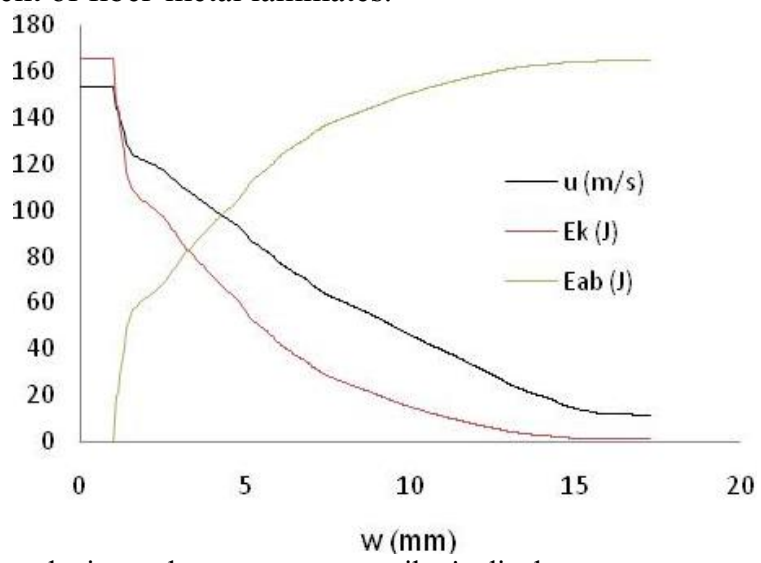

Fig. 4. Striker-target velocity and energy versus striker's displacement curves of a 1080 steel based 3/2-0.5 fiber-metal laminate subjected to ballistic impact by a cylindrical projectile.

Using numerous displacement contours of the projectile and the panel at characteristic stages of the ballistic impact phenomenon in combination with pertinent numerical results, it is revealed that during the beginning of the considered impact event, the variations of characteristic impact variables cannot be captured using a spring-mass model with a single degree of freedom. At least two degrees of freedom are needed for the mechanical system. A single degree of freedom mechanical system may be used in order to simulate the impact event satisfactorily after the beginning, when the local indentation of the panel under the projectile is completed, until the perforation of the panel.

\section{Conclusions}

This article deals with the transient response of square clamped fiber-metal laminates and monolithic metal plates subjected to central normal ballistic impact by a rigid flat-faced 
cylindrical projectile. ANSYS LS-DYNA explicit nonlinear finite element software is employed in order to simulate the ballistic impact phenomenon.

Using the five validated finite element models of GLARE 5-2/1-0.5, GLARE 5-3/2-0.3 and GLARE 5-3/2-0.5 fiber-metal laminates, and 2024-T3 monolithic aluminum plates with $1.6 \mathrm{~mm}$ and $3.2 \mathrm{~mm}$ thickness, the ballistic impact response of steel based fiber-metal laminates and monolithic plates is studied. Six steel alloys are examined, namely 304 stainless steel, 1010, 1080, 4340, A36 steel and DP 590 dual phase steel.

It is found that the ballistic limits of the panels can be substantially affected by the constituent metal alloy. The stainless steel based panels offer the highest ballistic resistance followed by the A36 steel based panels which in turn have higher ballistic resistance than the 2024-T3 aluminum based panels. The A36 steel based panels have higher ballistic limit than the 1010 steel based panels which in turn have higher ballistic limit than the 1080 steel based panels. The 2024-T3 aluminum, the 1080 steel and the DP 590 dual phase steel based panels have comparable relatively low ballistic resistance.

It is revealed that during the beginning of the considered impact phenomenon the variations of characteristic impact variables cannot be captured using a spring-mass model with a single degree of freedom. At least two degrees of freedom are needed for the mechanical system. A single degree of freedom mechanical system may be used in order to simulate the impact event satisfactorily after the beginning, when the local indentation of the panel under the projectile is completed, until the perforation of the panel.

\section{References}

1. Q. Liu, J. Ma, L. Kang, et al, Mater. Des. 88, 643 (2015)

2. R.E. Farsani, S.M.R. Khalili, V. Daghigh, Int. J. Damage Mech. 23, 729 (2014)

3. V. Daghigh, S.M.R. Khalili, R.E. Farsani, Comp.: Part B 91, 275 (2016)

4. T. Parnanen, M. Kanerva, E. Sarlin, et al, Comp. Struct. 119, 777 (2015)

5. E. Poodts, D. Ghelli, T. Brugo, et al, Comp. Struct. 129, 36 (2015)

6. N. Petkune, T. Donchev, H. Hadavinia, et al, Constr. Build. Mater. 117, 198 (2016)

7. S.W.F. Spronk, I. Sen, R.C. Alderliesten, Int. J. Fatigue 70, 428 (2015)

8. T. Parnanen, A. Vanttinen, M. Kanerva, et al, Appl. Compos. Mater. 23, 1151 (2016)

9. T. Nowak, J. Schmidt, Comp. Struct. 131, 637 (2015)

10. M.S. Hoo Fatt, C. Lin, D.M. Revilock jr, et al, Comp. Struct. 61, 73 (2003)

11. LS-DYNA, Keyword user's manual (Livermore Software Technology Corporation, 2014)

12. LS-DYNA Support, http://www.dynasupport.com

13. G.S.E. Bikakis, C.D. Dimou, E.P. Sideridis, Aerosp. Sci. Technol. 69, 201 (2017)

14. T.P. Vo, Z.W. Guan, W.J. Cantwell, et al, Comp.: Part B 44, 141 (2013)

15. J. Dean, C.S. Dunleavy, P.M. Brown, et al, Impact Eng. 36, 1250 (2009)

16. AZO MATERIALS, http://www.azom.com

17. N.S. Brar, V.S. Joshi, B.W. Harris, AIP Conference Proceedings 955, 627 (2007)

18. J.D. Cinnamon, A.N. Palazotto, N.S. Brar, et al, AIP Conference Proceedings 845, 709 (2006)

19. G.R. Johnson, W.H. Cook, Engng. Fracture Mech. 21, 31 (1985)

20. L. Schwer, 6th European LS-DYNA users' conference, (2007)

21. AKSTEEL, http://www.aksteel.com

22. K. Vedantam, D. Bajaj, N.S. Brar, et al, AIP Conference Proceedings 845, 775 (2006)

23. R.S.J. Corran, P.J. Shadbolt, C. Ruiz, Impact Eng. 1, 3 (1983)

24. G.G. Corbet, S.R. Reid, W. Johnson, Impact Eng. 18, 141 (1996) 